\title{
NIVEL DE CONTAMINACIÓN ENTEROPARASITARIA DE LECHUGAS (Lactuca sativa) IRRIGADAS CON AGUAS DEL RÍO RÍMAC PARA CONSUMO HUMANO EN LA ZONA DE CARAPONGO.
}

\author{
Félix Nicolás PALACIOS MORALES ${ }^{1}$
}

\begin{abstract}
RESUMEN
Objetivo: El presente estudio tuvo como objetivo determinar el nivel de la contaminación enteroparasitaria de lechugas para consumo humano irrigadas con aguas servidas del río Rímac en poscosecha. Metodología: Se estableció tres zonas de estudio Z-1 curva del cerro Cuncacucho, Z-2 puente o mercado de Carapongo a $50 \mathrm{~m}$ de rivera del río y Z-3 paradero Portillo a $50 \mathrm{~m}$ de la ribera del río Las muestras de lechuga, (Lactuca sativa), fueron tomadas in situ en número de dos unidades, de las cuales se utilizaron aproximadamente $200 \mathrm{~g}$. de hojas basales que estuvieron en contacto directo con el suelo y las aguas durante el período de irrigación; la colecta se hizo mediante el uso de guantes de examen, bisturíes, pinzas simples y colectadas en bolsas cristal de polietileno con click de seguridad.Las muestras fueron procesadas por el método directo de observación y por la técnica de coloración de Ziehl Neelsen modificado. Resultados: Los resultados obtenidos demostraron un nivel de contaminación enteroparasitaria de lechugas, encontrándose los siguientes parásitos Blastocystis hominis (23.88\%), Balantidium coli (4.47\%), Entamoeba coli (1\%) Crytosporidium spp. (10.44\%), Giardia lamblia. (8.95\%), Ascaris lumbricoides. (17.91\%), entamoeba hystolitica (19.40\%), Isospora sp (2.98\%), toxocara sp (5.97\%). Frente a estos resultados, es necesario que se tomen medidas de control para mejorar la calidad higiénica sanitaria de estos alimentos. Por los resultados hallados en el presente estudio se recomienda el monitoreo continuo en pos cosecha de lechuga como producto hortícola de consumo directo. Conclusiones: Se encontró la presencia de especies de enteroparásitos en las lechugas (Lactuca sativa) para consumo humano irrigadas con aguas servidas del río Rímac en la zona hortícola de Carapongo. El mayor porcentaje de enteroparásitos fueron Blastocystis hominis 29.91\%, Entamoeba hystolitica $19.03 \%$, Áscaris lumbricoides $17.84 \%$, Cryptosporidium s $11.14 \%$ respectivamente. Se ha podido evaluar la presencia de enteroparàsitos en varios niveles descritos como alto, medio, regular y bajo según los estándares de la OMS.
\end{abstract}

Palabras Clave: Protozoosis, cryptosporidium, isospora, giardia, lechuga.

\begin{abstract}
Objective: This study aimed to determine the enteroparasitosis pollution level from lettuce meant for human consumption irrigated with wastewater from the Rimac River in post-harvest season. Methodology: It were established three study sites: Z-1 Cuncacucho hill curve; Z-2 the bridge area or the Carapongo market, 50 meters from the river bank; and Z-3 around the bus stop The Portillo 50 meters away from the river bank. The lettuce samples (Lactuca sativa) were taken in situ in the number of two lettuce heads from which it was used approximately 200 grams of basal leaves which were in direct contact with the soil and water during the irrigation period. The collection was done through the use of examination gloves, scalpels, simple tweezers and the samples were collected in polyethylene crystal bags with security locks. The samples were processed by the direct method of observation and by the modified Ziehl Neelsen coloring technique. Results: The results showed that there were enteroparasitosis contamination levels in the lettuce leaves with the following parasites: blastocystis hominis (23.88\%), balantidium coli (4.47\%), entamoeba coli (1\%), Cryptosporidium spp. (10.44\%), Giardia lamblia. (8.95\%), Ascaris lumbricoides (17.91\%), Entamoeba hystolitica (19.40\%), Isospora sp (2.98\%), Toxocara sp (5.97\%). Given these results, it is necessary to take control measures to improve the hygienic health quality for these foods. From the results found in this study it
\end{abstract}

\footnotetext{
${ }^{1}$ Ingeniero Forestal, Jefe de Laboratorio de Biología de la Facultad de Ciencias de la Salud de la Universidad Peruana Unión.
} 
is recommended a continuous monitoring in the lettuce post-harvest procedure for being a horticultural product for direct consumption. Conclusions: It was found the presence of species of enteroparasites in lettuce (Lactuca sativa) for human consumption irrigated with sewage water that comes from Rimac River in the Carapongo horticulture area. The highest percentage of enteroparasites were Blastocystis hominis 29.91\%, Entamoeba hystolitica 19.03\%, Ascaris lumbricoides $17.84 \%$, Cryptosporidium s $11.14 \%$ respectively. It was possible to assess the presence of enteroparasites in various levels described as high, medium, regular and low by the WHO standards.

Key Words: Protozoosis, Cryptosporidium, Isospora, Giardia, lettuce.

\section{INTRODUCCIÓN}

La agricultura urbana es la actividad centrada en la producción agrícola de hortalizas, frutales, plantas medicinales, ornamentales, etc. y pecuarios (animales menores) para autoconsumo o comercialización. Al mismo tiempo el crecimiento poblacional ha incrementado los sistemas de aguas residuales y son fuente de contaminación para el ser humano, los animales domésticos e incluso para animales silvestres, como ocurre en ambas márgenes del río irrigadas por afluentes que ingresan como aguas servidas al lecho del río Rímac.. (Vilcamiche, Z. Romero, S. Ango, H. 2006). Las aguas residuales son portadoras de una amplia variedad de microorganismos patógenos entre los que se encuentran los protozoarios y los helmintos que parasitan al hombre y a los animales domésticos, ya que dichos parásitos son evacuados con las heces al cauce del río, estas aguas son utilizadas por los horticultores de Carapongo y generan protozoosis intestinales transmitidas por alimentos más comunes que afectan al hombre y son las producidas por: Giardia sp, Entamoeba histolytica, Entamoeba coli, Cryptosporidium parvum e Isospora sp., que son transmitidas primariamente por vía fecal-oral (Motarjemi y col., 1994; Murga-Gutiérrez, 1995); Asimismo, es el medio de contaminación de las verduras consumidas crudas, como un factor en la diseminación de enteroparásitos, debido a que los campos de cultivo son irrigados con aguas servidas (Franjola y Gutiérrez, 1984; Herrera y Obeso, 1987; Murga-Gutiérrez, 1995).

Según los padrones de uso agrícola de las Juntas de Usuarios de los ríos Chillón, Rímac y Lurín, actualmente se encuentran bajo riego en Lima Metropolitana 12,680 ha, pertenecientes a 7,601 agricultores organizados en 35 comisiones de regantes de los cuales la cuenca del Rímac tiene 11 juntas y 3958 ha. de suelos agrícolas bajo riego y 1922 usuarios.

El crecimiento urbano y periurbano ha disminuido las tierras agrícolas de uso mayor y de buena calidad de Lima Metropolitana de $600 \mathrm{Km} 2 \quad$ (98\% del área total) a $125 \mathrm{Km} 2(21 \%$ del área total). Los sistemas de desagüe vierten sus aguas servidas al lecho del río Rímac, cuenca principal que es utilizada por el sistema de irrigación en la margen izquierda y derecha del mismo valle; éste lleva en su cauce aguas servidas que infectan con enteroparásitos las hortalizas de consumo directo irrigadas en la zona de Carapongo. En el presente estudio se estableció tres zonas de estudio Z-1 curva del cerro Cuncacucho, Z-2 puente o mercado de Carapongo a $50 \mathrm{~m}$ de rivera del río y Z-3 paradero Portillo a $50 \mathrm{~m}$ de rivera del río. Para determinar la contaminación de lechugas con enteroparásitos se tomaron 75 muestras en pre y poscosecha. Las muestras de lechuga (Lactuca sativa), fueron tomadas in situ al azar en número de dos unidades por zona y llenadas en bolsas cristal estéril, etiquetada y rotulada y fueron trasladadas al laboratorio, de los cuales se utilizaron aproximadamente $200 \mathrm{~g}$. de hojas basales que estuvieron en contacto directo con el suelo y las aguas durante el periodo de irrigación. El método de obtención y procesamiento de la muestras para su observación e identificación microscópica fue mediante el lavado por fricción en frasco de boca ancha de $500 \mathrm{ml}$ con agua destilada estéril. Se agitó y se dejó en reposo por 24 horas. Se retiraron las hojas para decantar los elementos pesados por una hora, luego separamos el precipitado del sobrenadante tomando 9/10 partes y colocadas en tubos de centrifugación de $15 \mathrm{ml}$ cada uno y centrifugados por 10 min. a 3000 rpm. Se diluyó con solución salina a $0.85 \%$ y se agregó lugol para su observación y descripción microscópica. En este proceso se encontró los siguientes microorganismos de las zonas de evaluación en promedio: Blastocystis hominis (23.61\%), Áscaris lumbricoides (17.84\%), Entamoeba hystolitica (19.03\%), Cryptospridium sp $(11.14 \%)$, Giardia lamblia $(7.88 \%)$, Toxocara sp (6.96\%), Balantidium coli (5.37\%), Isospora sp $(1.85 \%)$. Por lo tanto, el presente estudio tuvo como finalidad determinar el nivel la contaminación enteroparasitaria de lechugas para consumo humano irrigadas con aguas servidas del río Rímac en la zona agrícola de Carapongo.

\section{MATERIALES Y MÉTODOS}

Las muestras de lechuga (Lactuca sativa) se tomaron in situ tanto en el período de pre y poscosecha de 
terrenos elegidos al azar en la zona agrícola de carapongo comprendida en tres zonas de muestreo: Curva del Cerro Cuncacucho, Puente y San Antonio de Carapongo. El período de muestreo estuvo comprendido entre los meses de marzo a setiembre de 2009, a una temperatura promedio de $22{ }^{\circ} \mathrm{C}$.

Se obtuvo una muestra previa de 45 cabezas de lechuga y 15 por zona, para la determinación del tamaño muestral debido a que no hubo estudios previos zonificados. La prevalencia de la muestra mostró un 5\% de contaminación parasitaria, procedente de la zona de estudio. Con el fin de determinar el tamaño de la muestra se utilizó la fórmula para estimar una proporción (Daniel, 1996).

$$
\mathrm{n}=\frac{\mathrm{Z}^{2} \cdot \mathrm{p} \cdot \mathrm{q}}{\mathrm{E} 2}
$$

Donde:

$\mathrm{n}=$ Tamaño muestra

$Z=95 \%=1.96$ (Nivel de confianza).

$\mathrm{p}=0.05$ (proporción hallada en la muestra previa).

$\mathrm{q}=1-\mathrm{p}=0.95$

$\mathrm{E}=0.05$ (precisión).

$$
\begin{aligned}
& n=72.9904 \\
& n=73
\end{aligned}
$$

Se estableció tres zonas de estudio Z-1 curva del cerro Cuncacucho, Z-2 puente o mercado de Carapongo a 50 $\mathrm{m}$ de ribera del río y Z-3 paradero Portillo a 50 m de ribera del río. Las muestras de lechuga (Lactuca sativa), fueron tomadas in situ en número de dos unidades de los cuales se utilizaron aproximadamente $200 \mathrm{~g}$. de hojas basales que estuvieron en contacto directo con el suelo y las aguas durante el período de irrigación. La colecta se hizo mediante el uso de guantes de examen, bisturíes, pinzas simples y colectadas en bolsas cristal de polietileno con click de seguridad. Las muestras fueron etiquetadas usando una numeración correlativa por zona, sellándose y depositándose en conservadores con hielo para su transporte hacia el Laboratorio de Biología de la Facultad de Ciencias de la Salud de la Universidad Peruana Unión.

En el Laboratorio, cada muestra fue depositada en un beaker de litro conteniendo $300 \mathrm{ml}$ de agua destilada estéril, se procedió a lavar por fricción por un tiempo aproximado de 2 minutos para luego ser tamizada. Se utilizó la técnica de sedimentación con la dilución obtenida previo lavado, tamizado y en reposo a temperatura ambiente por 24 horas al término del cual se realizó el centrifugado para su respectivo estudio.
Se evaluó las formas parasitarias, presencia de quistes mediante el método de observación directa del sedimento, adicionándole lugol o azul de metileno para su mejor observación, ya que el citoplasma presenta afinidad tintoreal (Agurto, 1983). Se realizaron dos extensiones del sedimento obtenido que posteriormente serán coloreados con la técnica de Ziehl Neelsen modificado, para la observación de Cryptosporidium parvum (Henricksen y Pohlenz, 1981). Los ooquistes de Isospora sp. fueron diagnosticados por la observación directa del sedimento.

\section{A. Reactivos:}

a) Fucsina básica fenicada: 8 g de fucsina básica fenicada en $345 \mathrm{ml}$ de agua destilada caliente. A esta solución se le agregó $30 \mathrm{ml}$ de fenol líquido, homogeneizándose y filtrándose antes de usar.

b) Verde malaquita: $5 \mathrm{~g}$ de verde malaquita en 100 $\mathrm{ml}$ de agua destilada, filtrándose antes de usar.

c) Ácido sulfúrico al 2\%: $13 \mathrm{ml}$ de ácido sulfúrico en agua hasta completar $100 \mathrm{ml}$ de solución.

\section{B. Procedimiento.}

La colección de muestras lechugas fue realizada en tres zonas (Z-1, Z-2 y Z-3) de 25 unidades cada una realizadas en distintos tiempos y por dos unidades de muestreo por vez, éstas fueron colocadas en bolsas cristal estériles tipo “click”, etiquetadas y registradas su procedencia. En caso de no ser analizadas de inmediato en el laboratorio, se mantuvieron en refrigeración hasta su procesamiento por un tiempo no mayor a 24 horas.

Para el análisis parasitológico, las lechugas fueron sometidas a la metodología de Álvarez y cols, con las siguientes modificaciones: las lechugas fueron deshojadas una por una, luego sumergidas en su totalidad en agua destilada estéril, contenida en envases de vidrio previamente esterilizadas. Las muestras de lechuga se lavaron por fricción y fueron dejadas en reposo por 24 horas, para luego de este tiempo retirarlas y dejarlas en el agua de nuevo en reposo por 1 hora.

Finalmente, se decantó con cuidado las 3/4 partes de la solución, de modo que la porción fina de aproximadamente $35 \mathrm{ml}$. Este volumen se dispensó a un tubo de ensayo grande para ser centrifugado por 10' de 2.500 - 3.000 r.p.m. Posteriormente a la centrifugación se descartó el sobrenadante y el sedimento fue analizado mediante un montaje en un portaobjetos con su respectivo cubreobjetos y observados a 10X y 40X. En caso de observarse quistes protozoarios se realizaba el montaje con lugol. 


\section{Lectura de láminas}

$\mathbf{1}^{\mathbf{0}} \mathrm{El}$ diagnóstico comprendió la lectura de la muestra con un aumento de 400X. Se logró describir las características de los parásitos que se encontrasen al menos siendo visibles las características morfológicas descritas (Feldman y col., 1992).

$2^{\circ}$ El Diagnóstico de Isosporas sp, fueron observadas a 100X, designándose como positivas luego de su esporulación al medio ambiente, apreciándose todas las características del ooquiste ya mencionadas (Atías,1991). $3^{\mathbf{0}}$ El diagnóstico de Cryptosporidium parvum fue realizado mediante un aumento de $400 \mathrm{X}$, y para confirmar las muestras positivas, se observò a 1000X.

Se visualizaron como formas esféricas, de color rojo a fucsia encendido con presencia de granulaciones oscuras en su interior, contrastando con el tono verde del fondo. Las levaduras adquirieron coloraciones similares, diferenciándose de los quistes por sus características morfológicas, tintoriales, diversidad de tamaño y la tinción homogénea que presenta observándose una imagen plana (Henricksen y Pohlenz, 1981).

\section{RESULTADOS Y DISCUSIÓN}

En los artículos $56^{\circ}$ y $405^{\circ}$ del Código Latinoamericano de Alimentos, y el artículo $24^{\circ}$ del Reglamento de Control de Alimentos, declaran la inocuidad sanitaria de los alimentos que son consumidos crudos, como las lechugas se deberían evitar la presencia de formas parasitarias en las verduras de consumo directo.

Según el estudio, la lechuga es una hortaliza de consumo crudo y directo que presenta un alto nivel de contaminación enteroparasitaria por ser irrigada por inundación con aguas servidas del río Rímac, éstas quedan expuestas más tiempo al agua y su textura rugosa favorece mayor retención y adherencia en la superficie basal de sus hojas en comparación con otras verduras que son de tallos cortos; como rabanito, culantro, perejil, espinaca, berros, tomate, pepino, etc.

Tabla 1. Muestras de lechugas contaminadas con enteroparásitos según la zona de muestreo en pos cosecha.

\begin{tabular}{|c|c|c|c|c|}
\hline Zona de muestreo & $\begin{array}{c}\mathrm{N}^{\circ} \text { de lechugas } \\
\text { a analizar }\end{array}$ & $\begin{array}{l}\mathrm{N}^{\circ} \text { de lechugas } \\
\text { contaminadas }\end{array}$ & $\% \mathrm{M}+$ & Nivel \\
\hline Z-1 curva Cuncacucho & 24.33 & 23 & 31.5068 & \\
\hline Z-2 Puente Carapongo & 24.33 & 26 & 35.6164 & \\
\hline Z-3 San Antonio de Carapongo & 24.33 & 24 & 32.8767 & \\
\hline TOTAL & 72.99 & 73 & 99.999 & \\
\hline
\end{tabular}

De un total de 75 lechugas se tomaron muestras en tres zonas $(Z-1=23, Z-2=26$ y Z-3=24) y se encontró contaminación parasitaria en un $(31.50 \%),(35.61 \%)$ y $(32.87 \%)$ 
Tabla 2. Lechugas contaminadas por enteroparásitos identificados por zona de evaluación pos cosecha.

\begin{tabular}{|c|c|c|c|c|c|c|c|c|}
\hline \multirow{2}{*}{ Tipos de parásitos } & \multicolumn{2}{|c|}{ Z1 } & \multicolumn{2}{|c|}{$\mathbf{Z 2}$} & \multicolumn{2}{|r|}{$\mathbf{Z 3}$} & \multirow{2}{*}{$\%$} & \multirow{2}{*}{$\bar{X}$} \\
\hline & $\mathrm{n}$ & $\%$ & $\mathrm{n}$ & $\%$ & $\mathrm{n}$ & $\%$ & & \\
\hline Blastocystis hominis & 6 & 35.294 & 10 & 27.777 & 4 & 26.666 & & 29.91 \\
\hline Balantidium coli & 0 & 0 & 1 & 2.777 & 2 & 13.333 & & 5.37 \\
\hline Cryptosporidium sp & 2 & 11.764 & 3 & 8.333 & 2 & 13.333 & & 11.14 \\
\hline Giardia lamblia & 1 & 5.882 & 4 & 11.111 & 1 & 6.666 & & 7.88 \\
\hline Áscaris lumbricoides & 4 & 23.529 & 6 & 16.666 & 2 & 13.333 & & 17.84 \\
\hline Entamoeba hystolitica & 3 & 17.647 & 7 & 19.444 & 3 & 20.000 & & 19.03 \\
\hline Isospora sp & 0 & 0 & 2 & 5.555 & 0 & 0 & & 1.85 \\
\hline Toxocara sp & 1 & 5.882 & 3 & 8.333 & 1 & 6.666 & & 6.96 \\
\hline TOTAL & 17 & 99.998 & 36 & 99.996 & 15 & 99.997 & & \\
\hline
\end{tabular}

En la tabla 2 se observa que después de haber lavado las hojas basales por fricción y se las dejó en reposo para decantar la solución por 24 hrs. Se separó el sobrenadante y el precipitado se observó con tinción Ziehl Neelsen, realizado el centrifugado. Se observaron al microscopio las siguientes especies tomadas en promedio de la tres zonas de estudio se encontró especies parasitarias propias del phylum Protozoo como sigue: Blastocystis hominis con un $29.91 \%$, Entamoeba hystolitica con 19.03\%, Áscaris lumbricoides 17.84\%, Cryptosporidium sp 11.14\%, Toxocara sp $6.96 \%$, Balantidium coli con $5.37 \%$ Giardia lamblia con $7.88 \%$, Isospora sp con un $1.85 \%$.

Tabla 3. Porcentaje de especies de enteroparásitos encontrados en muestras de lechuga y nivel de contaminación pos cosecha.

\begin{tabular}{lccc}
\multicolumn{1}{c}{ Zona de muestreo } & $\begin{array}{c}\mathrm{N}^{\circ} \text { de lechugas } \\
\text { a analizar }\end{array}$ & $\begin{array}{c}\mathrm{N}^{\circ} \text { de lechugas } \\
\text { contaminadas }\end{array}$ & $\% \mathrm{M}+$ \\
Blastocystis hominis & 20 & 29.411 & Alto \\
Balantidium coli & 3 & 4.411 & bajo \\
Cryptosporidium sp & 7 & 10.294 & Regular \\
Giardia lamblia & 6 & 8.823 & bajo \\
Áscaris lumbricoides & 12 & 17.647 & Medio \\
Entamoeba hystolitica & 13 & 19.117 & Medio \\
Isospora sp & 2 & 2.941 & Bajo \\
Toxocara sp & 5 & 7.352 & bajo \\
Total & 68 & 99.996 &
\end{tabular}

En la tabla $N^{\circ} 3$ según el porcentaje de las especies enteroparasitarias encontradas se puede observar la especie Blastocystis hominis con 29.41\% alcanza un nivel alto de contaminación, Cryptosporidium sp con 10.29\% nivel regular, Áscaris lumbricoides y Entamoeba hystolitica con $17.64 \%$ y 19.11\% respectivamente tienen un nivel medio de contaminación y Balantidium coli con $4.41 \%$, Giardia lamblia con 8.82\%, Isospora sp con 2.94\% y Toxocara sp con $7.35 \%$ presentan un nivel bajo de contaminación.

\section{CONCLUSIONES}

Se encontró la presencia de especies de enteroparásitos en las lechugas (Lactuca sativa) para consumo humano irrigadas con aguas servidas del río Rímac en la zona hortícola de Carapongo.
El mayor porcentaje de enteroparásitos fueron Blastocystis hominis 29.91\%, Entamoeba hystolitica 19.03\%, Áscaris lumbricoides 17.84\%, Cryptosporidium $\mathrm{s} 11.14 \%$ respectivamente. 
Se ha podido evaluar la presencia de enteroparásitos en varios niveles descritos como alto, medio, regular y bajo según los estándares de la OMS.

Las lechugas para consumo humano desde la precosecha y pos cosecha tienen dos fuentes de contaminación el primero son los períodos de riego por inundación donde las hojas adhieren los huevos de ooquistes en su superficie basal de las hojas y el segundo cuando las lechugas son lavadas para su mercadeo con las aguas de las acequias que son vertederos fecales de las viviendas contiguas a las acequias. Se deben establecer mecanismos de control y prevención respecto al pro- cesamiento de alimentos, en especial de verduras de consumo crudo.

La Organización Mundial de la Salud (OMS) ha establecido estrictos estándares para el rehúso de las aguas servidas que tengan como finalidad la irrigación de cultivos, indicando que el número de huevos de helmintos (Áscaris, Trichuris y Ancylostomoideos) en los efluentes, debe ser de uno o menos por litro de agua; sin embargo encontramos una sobrecarga de enteroparasitos que infectan a las hortalizas producidas en Carapongo por recibir doble dosis en pre y pos cosecha por tanto están sobrepasando los límites permisibles de estos estándares.

\section{REFERENCIAS BIBLIOGRÁFICAS}

1. Atencia, G. Navarro, J. Guitton, W. Cairo, Y. Chávez, M.. Prevalencia de Giardiasis Intestinal. Res. IV Congreso Peruano de Parasitología. Lima Perú; 2000. 44p.

2. Acha, NP. Szyfres, B.. Zoonosis y enfermedades transmisibles comunes al hombre y los animales. 2th. Ed.: 585-88, 611; 1989.13p.

3. Álvarez, M; Colina, M. y Rodríguez, H. Recuperación de formas evolutivas de enteroparásitos en legumbres del mercado Las Pulgas, Maracaibo, Venezuela. (Tesis de grado). La Universidad del Zulia. Facultad de Medicina. Escuela de Bioanálisis. Maracaibo, Venezuela; 1981:14.

4. Báez Abreu de Borges, F. Urquiola, G. Urrestarazu, M. Campo - Aasen, I. Serrano, N. Carvajal, Z. Ascanio, Y.. Etiopatogenie de las diarreas infecciosas crónicas en el adulto. Res. XI Congreso Latinoamericano de Parasitología. I Congreso Peruano de Parasitología. Lima - Perú; 1993. 88p.

5. Barboza, K.C.; Chirinos, C.J.; Mieres, M.V. y Pérez, N.C. Caracterización parasitológica de aguas residuales. (Tesis de grado). La Universidad del Zulia. Facultad de Medicina. Escuela de Bioanálisis. Maracaibo, Venezuela; 1997:58.

6. Baruffaldi, R.; Vessoni, T.C.; Machoshvili, I.A., ABE, L.C. Tratamiento químico de hortalizas poluidas. Rev. Saúde Publ. Sao Paulo; 1984: 225-234.

7. Cortéz, L. Cristóbal, O. Medina, S. Ayala, J.. Giardiasis en niños atendidos en el Hospital Sergio E. Bernales. Res. IV Congreso Peruano de Parasitología. Lima - Perú; 2000. 62p.
8. Chirinos, R. Galán, W, Villar, A. Lujan, M. Farfán, S. 2000. Descarte de parasitosis intestinal en pobladores del VI sector de Villa El Salvador. Res. IV Congreso Peruano de Parasitología. Lima - Perú; 2000. 51p.

9. Documento: Evolución de riesgos para la salud por el uso de aguas residuales en agricultura. Volumen I, Aspectos microbiológicos. Programa de Salud Ambiental. Organización Panamericana de la Salud, Organización Mundial de la Salud. Perú; 1990: 2:10.

10. Durán, E. Ortiz, J. Guzmán, G. Infantes, R. Villacaqui, R. Flores, V.. Enteroparasitosis en manipuladores de alimentos en el Distrito de Independencia - Huaraz. Res. IV Congreso Peruano de Parasitología. Lima-Perú; 2000. 61p.

11. Feldman, R. Del Valle, M. Gariboglio, M.. Detección de quistes de Giardia lamblia en agua. Serie Investigaciones Aplicada. Colección Hidrología No5. Consejo Federal de Inversiones. Buenos Aires - Argentina; 1992. 11-6p.

12. Feachmen, R.; Bradley, D.; Garelick, H. y Mara, D. Sanitation and disease; Health aspects of excreta and wastewater management. John Whiley \& Sons. New York; 1983. 501.

13. Franjola, R. y Gutiérrez, J. Estudio parasitológico en lechuga y beterragas en la ciudad de Valdivia, Chile. Rev. Méd. Chile; 1984. 112: 57-60p.

14. Frisancho, O.. Parasitosis Intestinal: Aspectos Fisiopatológicos. Rev. Gastroent; 1993. Per 13:45-9p. 
15. Herrera, J y Obeso, J.. Presencia de protozoarios y helmintos de interés sanitario en verduras expendidas en mercados de Lima Metropolitana [Tesis Farmacia y Bioquímica]. Facultad de Farmacia y Bioquímica: Universidad Nacional Mayor de San Marcos; 1987.

16. Juscamaita, CC. y Ango, AH. 2000. Asociaciones parasitarias frecuentes en niños menores de 5 años con desnutrición y aparentemente sanos - Ayacucho. Res. IV Congreso Peruano de Parasitología. Lima-Perú; 2000. 17p.

17. Kancha, S. Cuzcano, M. Recavarren, ME. Valderrama, DF.. Enteroparasitosis y Anemia en una población escolar de Quillabamba - La Convención, Cuzco. Res. IV Congreso Peruano de Parasitología. Lima-Perú; 2000. 26p.

18. Kageruka, P; Brand, J; Taelman, H and Jones, C. Modified Koster staining methad for the diagnosis of cryptosporidiosis. Am. Soc. Beige Med. Trop; 1984. 64: 171-175.

19. Motarjemi, Y. Käferstein, F. Moy, G. Quevedo, F.. Alimentos de destete contaminados: Un importante factor de riesgo de diarrea y malnutrición asociada. Bol. Oficina Sanit. Panam; 1994. 116(4) 313-27p.

20. Murga-Gutiérrez, S.. Formas parasitarias del hombre en Lactuca sativa "Lechuga”, cultivada en la provincia de Trujillo - Perú. Boletín Peruano de Parasitología; 1995. 11:42-45p.

21. Napolitano, J. and Coletti, C. Ocurrence of amoebae on Oak Leaf lettuce (Lactuca sativa var. crispa) and Boston lettuce (Latuca sativa var. Capitata). J. Protozool; 1984. 34(3): 454-455.

22. Palacios, L.M. Del Carmen, A. Castro, P. Lara, A. Caro, G. García, P.. Giardiasis: Prevalencia y сиаdro clínico en niños de Distrito de Masma Chicche. Res. IV Congreso Peruano de Parasitología. Lima - Perú; 2000. 11p.

23. Pillai, S.C. et. al. Sewage farming and sewage garden craps in relation to health. Bull. Nat. Inst. Sec. India; 1955. 10:160-179.
24. Poblete, L. y Ayaqui, R.. Parásitos intestinales en personas aparentemente sanas, Cuzco. Res. IV Congreso Peruano de Parasitología. Lima - Perú; 2000. 84p.

25. Recavarren, M. Caqui, E. Kancha, S. Cuzcano, M. Valderrama, D. 2000. Sensibilidad del Método parasitológico seriado en comparación con el simple. Res. IV Congreso Peruano de Parasitología. Lima - Perú; 2000. 22p.

26. Scott, J.C. Health and agriculture in china. Faber and Faber Ltd. London; 1953. 279.

27. Soliman, MM. Taghi-Kilani, R. Abou-Shady AF. El-Mageid SA. Handousa AA. Hegazi, MM. Belosevic, M.. Comparison of serum antibody responses to Giardia lamblia of symptomatic and asymptomatic patients. Am J Trop H y G 58(2); 1998. 232-239p.

28. Sotelo, N.. Giardisis en niños: aspectos clínicos y terapéuticos. Bol med of Hosp Infant Mex; 1998. 55(1): 47-53p.

29. Stien, J.L. and Schwartzbrod, J. Experimental contamination of vegetables with helminth eggs. Wat. Sic. Tech; 1990. 22(9): 51-57.

30. Tantaleán M. y Atencia G.. Nota sobre parasitismo intestinal diagnosticado en el IMT "Daniel A. Carrión”. Rev. Per. Med. Trop. U.N.M.S.M. 7(2); 1993. 99-3p.

31. Rude, RA. Survey of fresh vegetables for nematodes, amoebae ana Salmonella. J.A.O.A.C.. 67(3); 1984. 613-615.

32. Vilcamiche, Z. Romero, S. Ango, H.. Giardiasis en niños de 4 a 12 años de edad y su relación con algunos factores epidemiológicos, Ayacucho. Res. IV Congreso Peruano de Parasitología. Lima - Perú:; 2000. 21p.

33. Worth, R.M. Rural health in China. From village to commune. Amer. J. Hyg; 1963.77:228- 239.

34. Yoshiyama, M. Lau, D. Anderson, E. Odoñez, K. Figueroa, C.. Epidemiología de giardiasis en el Distrito de Lunahuana - Cañete. Res. IV Congreso Peruano de Parasitología. Lima - Perú; 2000. 32p. 